\title{
Top pair production at NNLO: an alternative approach
}

\author{
Sebastian Sapeta*t \\ Institute of Nuclear Physics, Polish Academy of Sciences \\ ul. Radzikowskiego 152, 31-342 Kraków, Poland \\ E-mail: sebastian.sapeta@ifj.edu.pl
}

We report on progress towards developing a framework for calculation of the NNLO cross section for top quark pair production. Our approach combines the $q_{T}$-slicing method and small- $q_{T}$ factorization, and its main technical challenge comes down to evaluation of the soft function at NNLO. We develop a framework based on sector decomposition and present a proof-of-concept calculation of the $n_{f}$ part of the NNLO soft function.

13th International Symposium on Radiative Corrections

24-29 September, 2017

St. Gilgen, Austria

\footnotetext{
* Speaker.

${ }^{\dagger}$ Based on work done in collaboration with Michał Czakon and René Ángeles-Martínez.
} 


\section{Introduction}

The increasing precision of the LHC data sets very high demands on the accuracy of theoretical predictions. The latter involve, in particular, corrections from higher perturbative orders of Quantum Chromodynamics. In this regard, the state of the art for most processes of interest has very recently changed from NLO to NNLO. Some gaps still need to be filled, however, and additional work is required to fully test the NNLO results and implement them in efficient public codes such that they can benefit experimental analyses. At the same time, the seminal calculation of the $\mathrm{N}^{3} \mathrm{LO}$ cross section for the Higgs boson production from gluon fusion [1] has marked the first step on the way towards pushing the accuracy of QCD predictions to the next level.

One of the most important processes measured at the LHC is the production of the top quark pair. It is relevant both in studies of properties of the Standard Model, as well as in searches for new physics, as it forms significant backgrounds to many signatures. The cross section for this process is currently known up to NNLO [2, 3, 4, 5, 6, 7].

In this proceedings, we present a proof-of-concept calculation of the $n_{f}$ part of the NNLO soft function for top quark pair production. This result, together with the framework and tools developed to obtain it, form key elements of an alternative calculation of the complete NNLO cross section for that process.

The motivation behind our work is two-fold. On one hand, it will lead to a second, independent result for the NNLO top pair production cross section, which, given the complexity of the calculation, is highly desirable. At the same time, our work forms a stepping stone towards developing a general framework for calculations of $\mathrm{N}^{3} \mathrm{LO} \mathrm{QCD}$ corrections to a wide range of processes of relevance for hadron colliders.

\section{Theoretical framework}

The approach for achieving NNLO accuracy for the top pair production, presented in this proceedings, is part of a wider strategy for calculating $\mathrm{N}^{m} \mathrm{LO}$ contributions to processes of the type

$$
h_{1}+h_{2} \rightarrow F\left(q_{T}\right)+X
$$

where two hadrons, $h_{1}$ and $h_{2}$, collide and produce an object $F$, which is registered in a detector, together with an undetected QCD radiation $X$. Our framework is suitable both when $F$, whose transverse momentum is denoted by $q_{T}$, is a colour-neutral object (single EW boson, pair of EW bosons, the Higgs) as well as when it carries colour, like in the case of the top quark pair.

We use the $q_{T}$-slicing method, which allows us to write the cross as a sum of two components, each of which is separately finite $[8,9]$

$$
\frac{\sigma_{\mathrm{N}^{m} \mathrm{LO}}^{F}}{d \Phi}=\int_{0}^{q_{T \text { cut }}} d q_{T} \frac{d \sigma_{\mathrm{N}^{m} \mathrm{LO}}^{F}}{d \Phi d q_{T}}+\int_{q_{T \text { cut }}}^{\infty} d q_{T} \frac{d \sigma_{\mathrm{N}^{m-1} \mathrm{LO}}^{F+\mathrm{jet}}}{d \Phi d q_{T}} .
$$

The advantage of this approach is that the second term in Eq. (2.2), which represents resolved emissions, is required only at the $\mathrm{N}^{m-1} \mathrm{LO}$ accuracy, and, in most interesting cases, it is already known. On the contrary, the first term, which combines virtual and unresolved real corrections, is usually unknown. However it is needed only in the small- $q_{T}$ approximation. 
In order to calculate the latter we use the Soft Collinear Effective Theory (SCET) [10], in which the cross section factorizes at small $q_{T}$ according to the formula

$$
\frac{d \sigma_{\mathrm{N}^{m} \mathrm{LO}}^{F}}{d \Phi d q_{T}}=\mathcal{B}_{1}^{\mathrm{N}^{m} \mathrm{LO}} \otimes \mathcal{B}_{2}^{\mathrm{N}^{m} \mathrm{LO}} \otimes \mathcal{H}^{\mathrm{N}^{m} \mathrm{LO}} \otimes \mathcal{S}^{\mathrm{N}^{m} \mathrm{LO}}+\mathcal{O}\left(\frac{q_{T}^{2}}{q^{2}}\right),
$$

where $q^{2}$ is an invariant mass of the object $F$. The functions appearing in the above equation account for contributions from different phase space regions of $X$. Specifically, the beam functions $\mathcal{B}_{i}$ sum up emissions of collinear and anti-collinear partons, the hard functions, $\mathcal{H}$, accounts for hard radiation, and the soft function, $\mathcal{S}$, sums emissions from soft, real gluons. Calculation of each of these functions is significantly simpler than calculation of the complete cross section. In addition, some of the functions are already available from literature.

In the case of the top quark pair production, the beam functions are known up to NNLO [11, 12] and the NNLO hard function can be extracted from Refs. [13, 14]. However, the small- $q_{T}$ soft function is only known up to NLO $[15,16]$. Hence, in order to achieve the NNLO accuracy of the $t \bar{t}$ cross section, one needs to calculate the missing NNLO correction to the soft function appearing in Eq. (2.3) (We note that such calculation shares many features with that of the NNLO soft function for boosted tops in the threshold limit [17]. However, the result for the latter is not of direct use in our context.)

The soft function for our process of interest can be schematically defined with the following equation

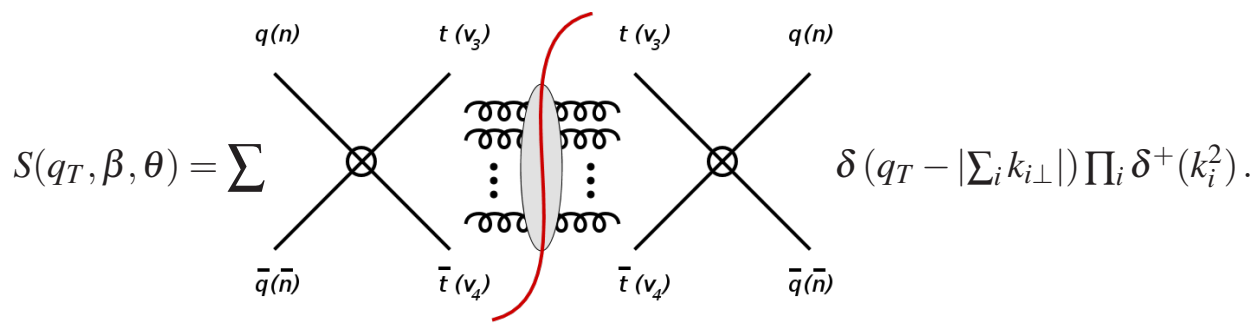

To this end, we focus on the $q \bar{q} \rightarrow t \bar{t}$ subprocess and introduce the following notation for the 4momenta: $p_{q}=m_{t} n, p_{\bar{q}}=m_{t} \bar{n}$ and $p_{t}=m_{t} v_{3}+l_{3}, p_{\bar{t}}=m_{t} v_{4}+l_{4}$, where $n=(1,0,0,1), \bar{n}=$ $(1,0,0,-1)$. We see that in the definition (2.4), the transverse momenta of real emissions are restricted to sum up to a fixed value of $q_{T}$. Because the gluons are soft, $l_{i} \ll m_{t} v_{i}$, and the velocities satisfy Born kinematics, $n+\bar{n}=v_{3}+v_{4}$, at each perturbative order. Apart from $q_{T}$, the soft function of Eq. (2.4) depends on $\beta=\sqrt{1-4 m_{t}^{2} / q^{2}}$ and $\theta$, where the latter is the scattering angle of the top quark in the $t \bar{t}$ rest frame.

The NNLO soft function corresponds to a sum of all $\mathcal{O}\left(\alpha_{s}^{2}\right)$ contributions from Eq. (2.4). They take forms of $2 d$-dimensional integrals which exhibit soft and rapidity singularities. The latter arise when the light-cone components of gluons 4-momenta become very small or very large, and are not removed by dimensional regularization. In our calculation, we adopt the prescription of Ref. [18] which turns the above divergences into poles in a new regulator $\alpha$. Even though the individual integrals suffer from rapidity divergences, their complete sum, hence the soft function, is finite in the limit $\alpha \rightarrow 0[15,18]$.

To calculate the soft function at this order, we designed the following integration strategy that can be algorithmically applied to evaluate each of its contributions. We start from analytically 
integrating 3 out of $2 d$ dimensions. Then, we map the remaining momenta into a unit hypercube (splitting the integral if necessary) and apply sector decomposition $[19,20]$ to disentangle overlapping singularities. Finally, we expand the result in $\alpha$ and $\varepsilon$ and numerically integrate the coefficients with help of the CUBA library [21].

\section{Proof of concept}

In order to validate our framework, we calculated the complete $n_{f}$ contribution to the NNLO soft function, which involves diagrams from Eq. (2.4) with one gluon on each side of the cut and the blob replaced with the quark loop.

The $n_{f}$ contribution has the advantage that it can be also obtained analytically. Integration over the quark loop momentum proceeds in a similar manner to the standard calculation of the vacuum polarization, only that now the tensor structure involves also the vector $n$, which is a consequence of introducing the rapidity regulator discussed above. One is then left with the integral over gluon momentum which we performed with help of the method of differential equations. The $n_{f}$ part of the NNLO soft function can be also singled out from the prediction of the Renormalization Group allowing for an additional cross check.

As mentioned in the previous section, all $\alpha$ poles have to cancel in the complete soft function. They also need to cancel within the subset of diagrams contributing to the $n_{f}$ part. And indeed, our analytic result contains contributions like

$$
\boldsymbol{w}_{13}^{q \bar{q}} I_{13}+\boldsymbol{w}_{24}^{q \bar{q}} I_{24}=\boldsymbol{w}_{13}^{q \bar{q}}\left(-\frac{8}{3 \varepsilon \alpha}+\frac{8}{3 \varepsilon \alpha}-\frac{8\left(5+3 \gamma_{E}+2 \ln 2\right)}{9 \alpha}+\frac{8\left(5+3 \gamma_{E}+2 \ln 2\right)}{9 \alpha}+\ldots\right)
$$

while from the numeric, sector decomposition-based approach, we obtain

$$
\boldsymbol{w}_{13}^{q \bar{q}} I_{13}+\boldsymbol{w}_{24}^{q \bar{q}} I_{24}=\boldsymbol{w}_{13}^{q \bar{q}}\left(-\frac{2.66597}{\varepsilon \alpha}+\frac{2.66597}{\varepsilon \alpha}-\frac{4.13986}{\alpha}+\frac{4.13986}{\alpha}+\ldots\right)
$$

In the above equations, $\boldsymbol{w}_{i j}$ and $I_{i j}$ correspond, respectively, to the colour matrix and the phase space integral parts of graphs in which the soft gluons are attached to the external lines $i$ and $j$, where we identify $(1,2,3,4) \leftrightarrow\left(n, \bar{n}, v_{3}, v_{4}\right)$.

As a second step of our validation, we checked that the result for the $n_{f}$ part of the NNLO soft function obtained through direct calculation with the strategy described in the preceding section reproduces exactly all terms predicted by the Renormalization Group (see [15, 17] for details), i.e. terms proportional to $\frac{1}{\varepsilon^{2}}, \frac{1}{\varepsilon}, \ln ^{2} \mu$ and $\ln \mu$.

As a final step in the proof of concept of our approach to calculation of the NNLO soft function for top pair production, we compared numerical results for combinations of quark-bubble graphs, obtained with the sector decomposition-based method, and the analytic results from the method of differential equations. An example comparison is presented in Fig. 1 (see Ref. [22] for another example) where points (lines) correspond to numeric (analytic) coefficients of different powers in the expansion in $\alpha$ and $\varepsilon$. We observe per-mille-level agreement for this and all the remaining graphs that contribute to the $n_{f}$ part of the NNLO soft function. 

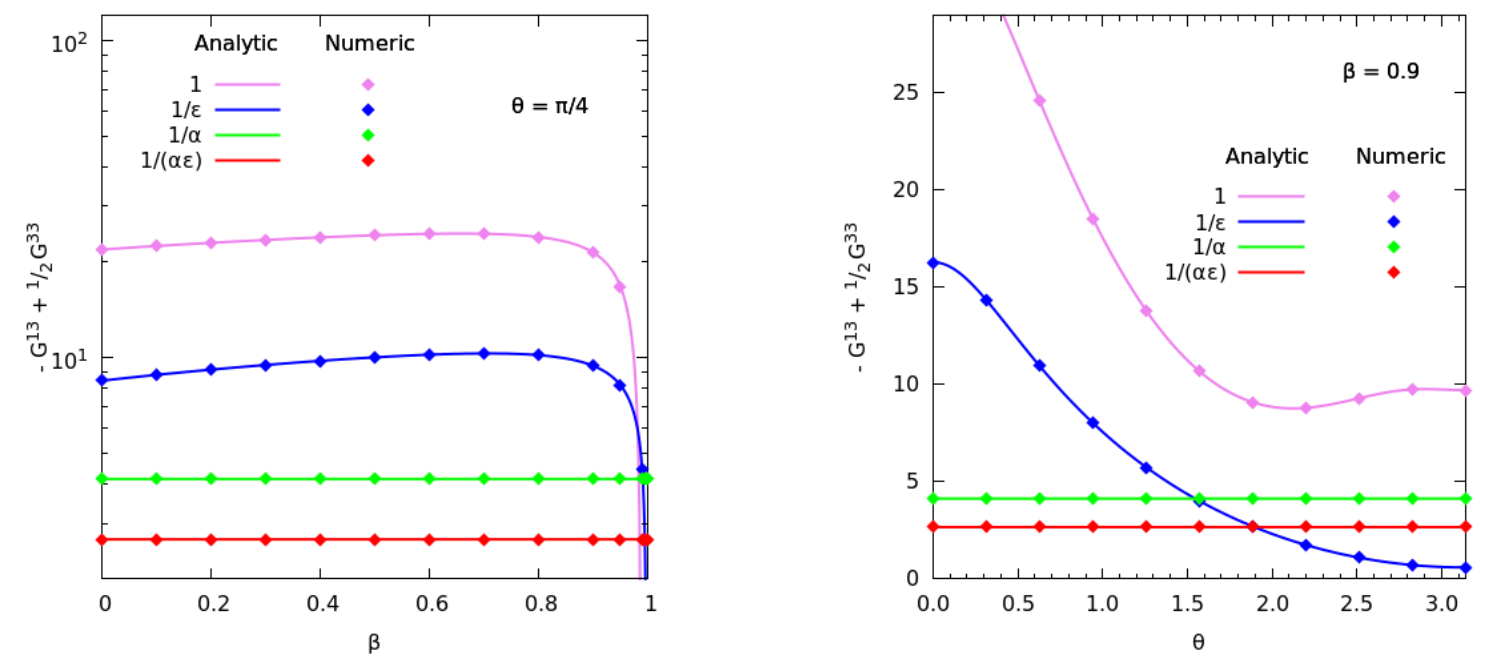

Figure 1: Comparison of numeric and analytic results for example graphs contributing to the $n_{f}$ part of the NNLO soft function. See text for details.

\section{Conclusions}

We presented a proof-of-concept calculation of the $\alpha_{s}^{2} n_{f}$ contribution to the small- $q_{T}$ soft function for top quark pair production. In order to evaluate the necessary divergent integrals we developed a framework based on sector decomposition. We performed a three-step validation of our results: by verifying that rapidity singularities cancel, by cross-checking that our direct calculation reproduces all terms predicted by the Renormalization Group and, finally, by finding a perfect agreement between numeric results from the sector decomposition-based framework and analytic results for individual graphs. The concepts and tools developed in our study allow for evaluation of all remaining integrals needed for calculation of the NNLO soft function and are generalizable to higher orders. Together with the $q_{T}$-slicing method, they offer a approach for calculation of the top quark pair production cross section at NNLO.

\section{Acknowledgements}

This work has been supported by the National Science Centre, Poland grant POLONEZ No. 2015/19/P/ST2/03007. The project has received funding from the European Union's Horizon 2020 research and innovation programme under the Marie Skłodowska-Curie grant agreement No. 665778. We are grateful to Mateusz Dobija for implementation of numerical integration with CUBA.

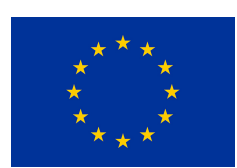

\section{References}

[1] C. Anastasiou, C. Duhr, F. Dulat, F. Herzog and B. Mistlberger, Phys. Rev. Lett. 114 (2015) 212001 doi:10.1103/PhysRevLett.114.212001 [arXiv:1503.06056 [hep-ph]].

[2] P. Bärnreuther, M. Czakon and A. Mitov, Phys. Rev. Lett. 109 (2012) 132001 doi:10.1103/PhysRevLett.109.132001 [arXiv:1204.5201 [hep-ph]]. 
[3] M. Czakon and A. Mitov, JHEP 1301 (2013) 080 doi:10.1007/JHEP01(2013)080 [arXiv:1210.6832 [hep-ph]].

[4] M. Czakon and A. Mitov, JHEP 1212 (2012) 054 doi:10.1007/JHEP12(2012)054 [arXiv:1207.0236 [hep-ph]].

[5] M. Czakon, P. Fiedler and A. Mitov, Phys. Rev. Lett. 110 (2013) 252004 doi:10.1103/PhysRevLett.110.252004 [arXiv:1303.6254 [hep-ph]].

[6] M. Czakon, D. Heymes and A. Mitov, Phys. Rev. Lett. 116 (2016) no.8, 082003 doi:10.1103/PhysRevLett.116.082003 [arXiv:1511.00549 [hep-ph]].

[7] M. Czakon, P. Fiedler, D. Heymes and A. Mitov, JHEP 1605 (2016) 034 doi:10.1007/JHEP05(2016)034 [arXiv:1601.05375 [hep-ph]].

[8] S. Catani and M. Grazzini, Phys. Rev. Lett. 98 (2007) 222002 doi:10.1103/PhysRevLett.98.222002 [hep-ph/0703012].

[9] R. Bonciani, S. Catani, M. Grazzini, H. Sargsyan and A. Torre, Eur. Phys. J. C 75 (2015) no.12, 581 doi:10.1140/epjc/s10052-015-3793-y [arXiv:1508.03585 [hep-ph]].

[10] T. Becher, A. Broggio and A. Ferroglia, Lect. Notes Phys. 896 (2015) pp.1 doi:10.1007/978-3-319-14848-9 [arXiv:1410.1892 [hep-ph]].

[11] T. Gehrmann, T. Lubbert and L. L. Yang, Phys. Rev. Lett. 109 (2012) 242003 doi:10.1103/PhysRevLett.109.242003 [arXiv:1209.0682 [hep-ph]].

[12] T. Gehrmann, T. Luebbert and L. L. Yang, JHEP 1406 (2014) 155 doi:10.1007/JHEP06(2014)155 [arXiv:1403.6451 [hep-ph]].

[13] M. Czakon, Phys. Lett. B 664 (2008) 307 doi:10.1016/j.physletb.2008.05.028 [arXiv:0803.1400 [hep-ph]].

[14] P. Bärnreuther, M. Czakon and P. Fiedler, JHEP 1402 (2014) 078 doi:10.1007/JHEP02(2014)078 [arXiv:1312.6279 [hep-ph]].

[15] H. T. Li, C. S. Li, D. Y. Shao, L. L. Yang and H. X. Zhu, Phys. Rev. D 88 (2013) 074004 doi:10.1103/PhysRevD.88.074004 [arXiv:1307.2464 [hep-ph]].

[16] S. Catani, M. Grazzini and A. Torre, Nucl. Phys. B 890 (2014) 518 doi:10.1016/j.nuclphysb.2014.11.019 [arXiv:1408.4564 [hep-ph]].

[17] A. Ferroglia, B. D. Pecjak, L. L. Yang, B. D. Pecjak and L. L. Yang, JHEP 1210 (2012) 180 doi:10.1007/JHEP10(2012)180 [arXiv:1207.4798 [hep-ph]].

[18] T. Becher and G. Bell, Phys. Lett. B 713 (2012) 41 doi:10.1016/j.physletb.2012.05.016 [arXiv:1112.3907 [hep-ph]].

[19] T. Binoth and G. Heinrich, Nucl. Phys. B 585 (2000) 741 doi:10.1016/S0550-3213(00)00429-6 [hep-ph/0004013].

[20] T. Binoth and G. Heinrich, Nucl. Phys. B 680 (2004) 375 doi:10.1016/j.nuclphysb.2003.12.023 [hep-ph/0305234].

[21] T. Hahn, Comput. Phys. Commun. 168 (2005) 78 doi:10.1016/j.cpc.2005.01.010 [hep-ph/0404043].

[22] R. Angeles-Martinez, Proceedings of the XXXIX International Conference of Theoretical Physics "Matter to the Deepest" 2017, Podlesice, Poland, Acta Phys. Polon. B (2017). 